\title{
Low-velocity impact response of nanotube-reinforced composite sandwich curved panels
}

\author{
WUNING MA, CHUNHAO YANG*®D, DAWEI MA and JIANLIN ZHONG \\ Department of Mechanical Engineering, Nanjing University of Science and Technology, Nanjing, China \\ e-mail: y674196@163.com
}

MS received 28 February 2019; revised 22 August 2019; accepted 9 September 2019

\begin{abstract}
The low-velocity impact response of the sandwich curved panels with functionally graded carbonnanotube-reinforced composite (FG-CNTRC) surface and isotropic foam core is discussed in this paper. Five types of stacking arrangements including uniform distribution of FG-CNTRC considering thermal environment were analysed. Using the Hertz contact law and rule of mixture model as well as the Kármán-type equations, the nonlinear formulations were built and solved by the two-step perturbation method. The carbon nanotubes' volume fraction, the structure size, the original impact velocity, temperature, relative thickness and the influence of gradient forms on the panels' impact behaviours were analysed. The outcomes show that the stiffness of the non-contact surface has large influence on contact response and various types of FG-CNTRC can be used for different operating conditions providing stiffness or cushion performance.
\end{abstract}

Keywords. Impact response; carbon nanotubes; functionally graded; sandwich curved panel; temperaturedependent.

\section{Introduction}

Since Iijima [1] discovered multi-walled carbon nanotubes (CNTs) in 1991, CNTs have gained great attention due to the unusual features, which are precious for optics, electronics and nanotechnology as well as other fields [2]. In the composite structure area, because of the thermo-stability, ultra-high elasticity modulus, big length-radius ratio and low density as well as fibroid structure, CNTs provide an ideal reinforcement [3-5]. It is helpful to make CNTs gradient configuration for improving the material's macro-mechanical performance with low content of CNTs.

Panels as a typical structure in the structural mechanics have been widely used in many fields, and the performance of functionally graded carbon-nanotubereinforced composite (FG-CNTRC) panels has been focused by researchers in recent years [6-14]. Aragh et al [15] studied the continuously graded CNT-reinforced cylindrical panels' vibrational behaviours on the basis of the Eshelby-Mori-Tanaka approach. Yas et al [16] explored FG-CNTRC cylindrical panel's vibrational features based on the three-dimensional theory of elasticity and found that the type of volume fraction and allocation of CNTs had significantly affected the

*For correspondence

Published online: 31 October 2019 normalized natural frequency. Shen and Xiang [17] analysed a shear-deformable FG-CNTRC cylindrical panel's big amplitude vibration behaviour by adopting a higher order shear deformation shell theory using the Mori-Tanaka model and Voigt model. Moreover, the outcomes ensured that Mori-Tanaka and Voigt models had the same accuracy to predict FG-CNTRC cylindrical panels' vibration features in a majority of cases. Zhang et al [18] analysed FG-CNTRC cylindrical panels' free vibration and flexural strength on the basis of the firstorder shear deformation shell theory. The effects of boundary conditions and edge-to-radius ratio as well as CNTs' volume fraction on free vibration responses and flexural strength were investigated. Moradi-Dastjerdi and Pourasghar [19] explored FG-CNTRC cylinder's dynamic response subjected to an effect load by messfree strategy. Lei et al [20, 21] discussed FG-CNTRC cylindrical panel's free vibration as well as dynamic stability under static and periodic axial force via the Ritz method. Sankar et al [22] studied the supersonic flutter features of FG-CNTRC shells as well as double curved sandwich panels on the basis of higher-order structural theory by adopting QUAD-8 shear flexible shell factor. They considered the effect of the CNT's volume fraction, radius-to-thickness ratio, the aspect ratio, the shell thickness and core-to-face sheet thickness as well as temperature on the flutter boundaries. Thomas and Roy [23] analysed the vibration and damping response of various kinds of shell structures of FG- 
CNTRC like cylindrical, doubly curved, ellipsoidal and spherical using Rayleigh damping patterns and finiteelement strategy. Shen and Xiang [24] presented a research on FG-CNTRC panels' post-buckling behaviours subjected to lateral pressure.

Since FG-CNTRC's impact response analysis gradually matured recently, scholars have researched lots of FGCNTRC structures' impact response [25-28]. Wang et al [29] discussed FG-CNTRC plates' low-velocity impact response on the basis of von Kármán nonlinearity as well as a high-order shear deformation theory. Song et al [30] studied FG-CNTRC plate's dynamic response subjected to the impact loading by Laplace transform and Duhamel integration as well as Fourier series expansion. Jam and Kiani [31] analysed FG-CNTRC beams' response subjected to the impacting mass by Ritz strategy and Timoshenko beam theory. Bayat et al [32] analysed FGCNTRC cylindrical shells' impact response on the basis of a higher-order shear deformation theory. The results showed that the shells' central deflection subjected to this influence had been sinusoidal. Malekzadeh and Dehbozorgi [33] analysed the low-velocity impact on the skew plate of FG-CNTRC by adopting the first-order shear deformation theory. It showed that the peak contact force increased but the impact duration decreased when the skew angle increased. Yang et al [34] discussed FGCNTRC laminates' low-velocity impact by adopting Kármán-type equations. The outcomes showed that the titanium alloy layer weakened FG-CNTRC's effect and improved the laminates' strength and stiffness. Song et al [35] studied aeroelastic FG-CNTRC panels' low-velocity impact behaviours in supersonic airflow. In addition, the aerodynamic pressure could be assessed by the first-order piston theory. Yang et al [36] performed FG-CNTRC spherical shells' low-velocity impact analysis by adopting Timoshenko-Midlin assumption for setting up the dimensionless nonlinear governing equations. The outcomes implied that the contact force had been decided collectively by both non-contact surface and contact surface's stiffness.

FG-CNTRC sandwich curved panels' low-velocity impact response, which consisted of two FG-CNTRC face sheets and a flexible core with thermal environment, has been considered in this paper. Five types of stacking arrangements including uniform distribution (UD) through the thickness direction have been analysed. The rule of mixture has been adopted for obtaining FG-CNTRC layer's material features. To study gradient forms' influence, relative thickness, temperature, initial impact velocity, structure size and CNT volume fraction, von-Kármán-type equations have been solved by a two-step perturbation technique used to describe the nonlinear formulations of the structure. The contact parameters are obtained using the Hertz contact law. The outcomes have enriched FG-CNTRC structures' theoretical research.

\section{Materials and methods}

\subsection{Material properties of the FG-CNTRC panel layer}

The rule of mixture model is used for defining the equivalent thermophysical parameters of FG-CNTRC in our research as mentioned in Ref. [17]. For exactly describing CNTs' gradient configuration, the properties of FGCNTRC layer can be defined as

$$
\begin{aligned}
E_{11} & =\eta_{1} V^{c n} E_{11}^{c n}+V^{m} E^{m} \\
\rho & =V^{c n} \rho^{c n}+V^{m} \rho^{m} \\
\frac{\eta_{2}}{E_{22}} & =\frac{V^{c n}}{E_{22}^{c n}}+\frac{V^{m}}{E^{m}}, \\
\frac{\eta_{3}}{G_{12}} & =\frac{V^{c n}}{G_{12}^{c n}}+\frac{V^{m}}{G^{m}} \\
\alpha_{11} & =\frac{V^{c n} E_{11}^{c n} \alpha_{11}^{c n}+V^{m} E^{m} \alpha^{m}}{V^{c n} E_{11}^{c n}+V^{m} E^{m}}, \\
v_{12} & =V_{*}^{c n} v_{12}^{c n}+V^{m} v^{m} \\
\alpha_{22} & =\left(1+v_{12}^{c n}\right) V^{c n} \alpha_{22}^{c n}+\left(1+v^{m}\right) V^{m} \alpha^{m}-v_{12} \alpha_{11}
\end{aligned}
$$

where $E_{11}^{c n}, E_{22}^{c n}$ and $G_{12}^{c n}$ are the elastic modulus and shear modulus of CNTs, respectively. $E^{m}$ and $G^{m}$ are the corresponding features of matrix material. The CNT's tangential direction is denoted by the subscript 11 , while the normal direction is denoted by the subscript $22 ; \eta_{j}(j=1,2,3)$ represents the efficiency parameter of CNTs. $V^{m}$ and $V^{c n}$ are, respectively, volume fractions of the matrix and CNTs, and they satisfy the criterion $V^{m}+V^{c n}=1 ; \rho^{c n}, \rho^{m}, \alpha_{11}^{c n}$, $\alpha_{22}^{c n}, \alpha^{m}, v_{12}^{c n}$ and $v^{m}$ are, respectively, the density, thermal expansion and Poisson's ratio of CNTs and matrix. CNTs' functionally graded profiles within the paper containing UD via the thickness direction have been explored, as presented in figure 1. The correlation volume fractions $V^{c n}$ for diversified graded profiles have been obtained by the following equations:
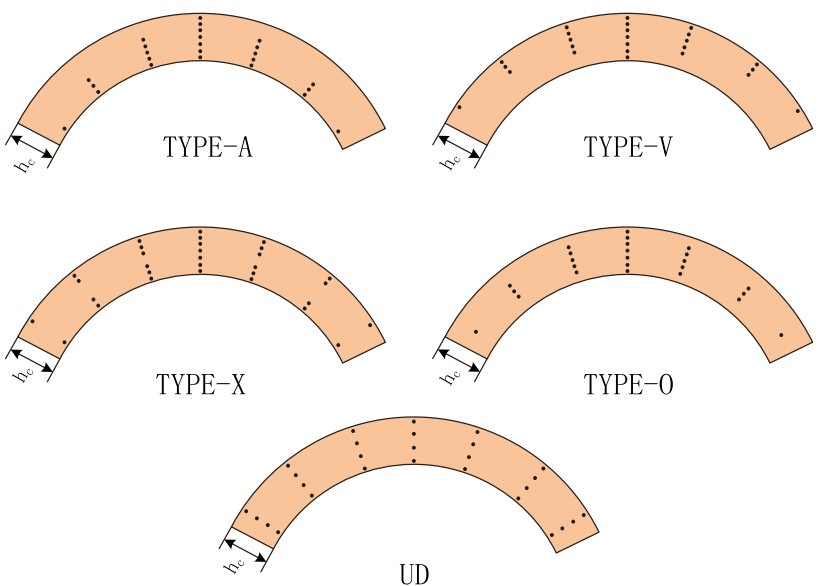

TYPE-0

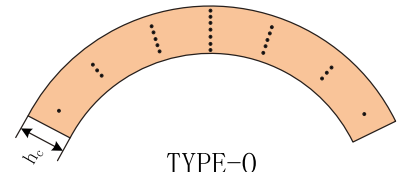

UD

Figure 1. Configuration of various CNTRC layers. 


$$
\begin{aligned}
& \text { TYPE - A: } V^{c n}=\left(1+\frac{2 z}{h_{c}}\right) V_{*}^{c n} \\
& \text { TYPE - V: } V^{c n}=\left(1-\frac{2 z}{h_{c}}\right) V_{*}^{c n} \\
& \text { TYPE - X: } V^{c n}=4\left(\frac{|z|}{h_{c}}\right) V_{*}^{c n} \\
& \text { TYPE - O: } V^{c n}=2\left(1-\frac{2|z|}{h_{c}}\right) V_{*}^{c n} \\
& \text { UD : } V^{c n}=V_{*}^{c n}
\end{aligned}
$$

where $z$ is the coordinate in the thickness direction and $h_{c}$ is FG-CNTRC layer's thickness. $V_{*}^{c n}$ can be evaluated as

$$
V^{c n}=\frac{m^{c n}}{m^{c n}+\left(\rho^{c n} / \rho^{m}\right)-\left(\rho^{c n} / \rho^{m}\right) m^{c n}}
$$

where $m^{c n}$ refers to CNTs' mass fraction.

\subsection{Calculation model of the curved panel}

Geometric parameters of the sandwich curved panel, made of FG-CNTRC and isotropic foam, are the two curvature radii $R_{1}$ and $R_{2}$, length $a$ and $b$, respectively, in the $X$ and $Y$ directions and thickness $h$ (FG-CNTRC layer $=h_{c}$, isotropic foam layer $=h_{f}$ ). The impactor's original velocity refers to $v_{0}$. The panel can be depicted by the right-hand coordinate system $(X, Y, Z)$ (as presented in figure 2). On the basis of the Timoshenko-Midlin assumption, each point's displacement parameters $\left(u_{1}, u_{2}, u_{3}\right)$ at a particular time and position on the panel can be assessed as [24]

$$
\begin{aligned}
& u_{1}= \bar{U}(X, Y, t)+Z \bar{\Psi}_{x}(X, Y, t) \\
& \quad+Z^{2} \xi_{x}(X, Y, t)+Z^{3} \zeta_{x}(X, Y, t) \\
& u_{2}= \bar{V}(X, Y, t)+Z \bar{\Psi}_{y}(X, Y, t) \\
& \quad \quad Z^{2} \xi_{y}(X, Y, t)+Z^{3} \zeta_{y}(X, Y, t) \\
& u_{3}=\bar{W}(X, Y, t)
\end{aligned}
$$

where $\bar{U}, \bar{V}$ and $\bar{W}$ refer to the displacement parameters on sandwich panel's mid-surface and $t$ is the time; $\bar{\Psi}_{x}$ and $\bar{\Psi}_{y}$ refer to the normal's rotations to the mid-surface about the $Y$ and $X$ axes, respectively. The stress function $F(X, Y)$ was

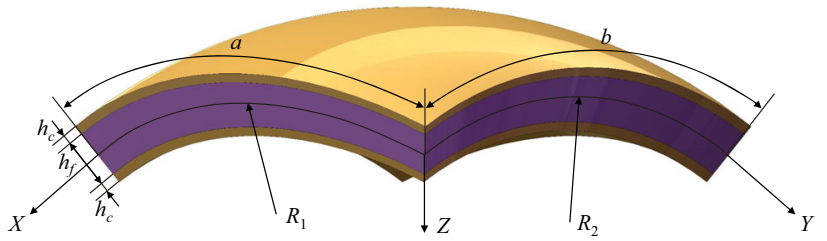

Figure 2. Geometric configuration of the FG-CNTRC sandwich curved panel. introduced to eliminate the displacement parameters [37]. The derivation process of the equations can be written in a form similar to that in Ref. [34], and the von-Kármán-type big deflection equations can be obtained as [24]

$$
\begin{aligned}
& L_{11}(\bar{W})-L_{12}\left(\bar{\Psi}_{x}\right)-L_{13}\left(\bar{\Psi}_{y}\right)+L_{14}(\bar{F}) \\
& \quad-L_{15}\left(\bar{N}^{T}\right)-L_{16}\left(\bar{M}^{T}\right) \\
& \quad-\frac{\bar{F}_{Y Y}}{R_{1}}-\frac{\bar{F}_{X X}}{R_{2}}=L\left(\bar{W}+\bar{W}^{*}, \bar{F}\right)+q \\
& L_{21}(\bar{F})+L_{22}\left(\bar{\Psi}_{x}\right)+L_{23}\left(\bar{\Psi}_{y}\right)-L_{24}(\bar{W})-L_{25}\left(\bar{N}^{T}\right) \\
& \quad+\frac{\bar{W}_{, Y Y}}{R_{1}}+\frac{\bar{W}_{, X X}}{R_{2}}=-L\left(\bar{W}+2 \bar{W}^{*}, \bar{W}\right) / 2 \\
& L_{31}(\bar{W})+L_{32}\left(\bar{\Psi}_{x}\right)-L_{33}\left(\bar{\Psi}_{y}\right)+L_{34}(\bar{F})-L_{35}\left(\bar{N}^{T}\right) \\
& \quad-L_{36}\left(\bar{S}^{T}\right)=0 \\
& L_{41}(\bar{W})-L_{42}\left(\bar{\Psi}_{x}\right)+L_{43}\left(\bar{\Psi}_{y}\right)+L_{44}(\bar{F})-L_{45}\left(\bar{N}^{T}\right) \\
& \quad-L_{46}\left(\bar{S}^{T}\right)=0
\end{aligned}
$$

where the operators $L()$ are depicted in detail in Appendix I. The high-order bending moment $\bar{P}_{i}$ and bending moment $\bar{M}_{i}$ as well as force $\bar{N}_{i}$ caused by diversified temperature $\Delta T(X, Y, Z)$ can be defined as

$$
\begin{aligned}
& {\left[\begin{array}{lll}
\bar{N}_{x}^{T} & \bar{M}_{x}^{T} & \bar{P}_{x}^{T} \\
\bar{N}_{y}^{T} & \bar{M}_{y}^{T} & \bar{P}_{y}^{T} \\
\bar{N}_{x y}^{T} & \bar{M}_{x y}^{T} & \bar{P}_{x y}^{T}
\end{array}\right]} \\
& =\sum_{i=1}^{N} \int_{h_{k-1}}^{h_{k}}\left(1, Z, Z^{3}\right)\left[\begin{array}{c}
A_{x} \\
A_{y} \\
A_{x y}
\end{array}\right] \Delta T(X, Y, Z) d Z \\
& {\left[\begin{array}{c}
\bar{S}_{x}^{T} \\
\bar{S}_{y}^{T} \\
\bar{S}_{x y}^{T}
\end{array}\right]=\left[\begin{array}{c}
\bar{M}_{x}^{T} \\
\bar{M}_{y}^{T} \\
\bar{M}_{x y}^{T}
\end{array}\right]-\frac{4}{3 h^{2}}\left[\begin{array}{c}
\bar{P}_{x}^{T} \\
\bar{P}_{y}^{T} \\
\bar{P}_{x y}^{T}
\end{array}\right]}
\end{aligned}
$$

where $\left[A_{x}, A_{y}, A_{x y}\right]^{T}$ can be defined as

$$
\left[\begin{array}{l}
A_{x} \\
A_{y} \\
A_{x y}
\end{array}\right]=-\left[\begin{array}{lll}
Q_{11} & Q_{12} & Q_{16} \\
Q_{12} & Q_{22} & Q_{26} \\
Q_{16} & Q_{26} & Q_{66}
\end{array}\right]\left[\begin{array}{ll}
1 & 0 \\
0 & 1 \\
0 & 0
\end{array}\right]\left[\begin{array}{l}
\alpha_{11} \\
\alpha_{22}
\end{array}\right] .
$$

Here, $Q_{i j}$ refers to the transformed elastic constant, where

$$
\begin{array}{r}
Q_{11}=\frac{E_{11}}{1-v_{12} v_{21}}, Q_{22}=\frac{E_{22}}{1-v_{12} v_{21}}, Q_{12}=\frac{v_{21} E_{11}}{1-v_{12} v_{21}} \\
Q_{16}=Q_{26}=0, Q_{44}=G_{23}, Q_{55}=G_{13}, Q_{66}=G_{12} .
\end{array}
$$

The boundary conditions can be set as simply supported. 


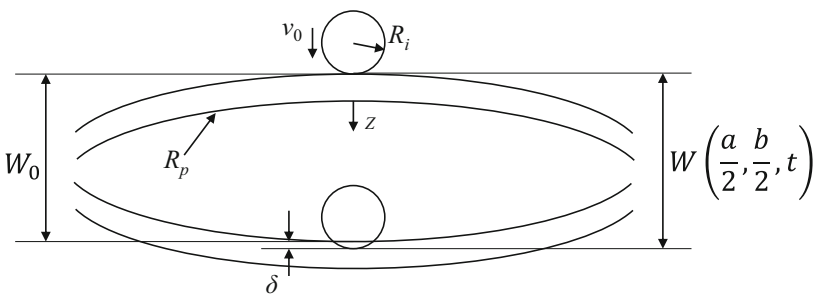

Figure 3. Deformation at the point of contact.

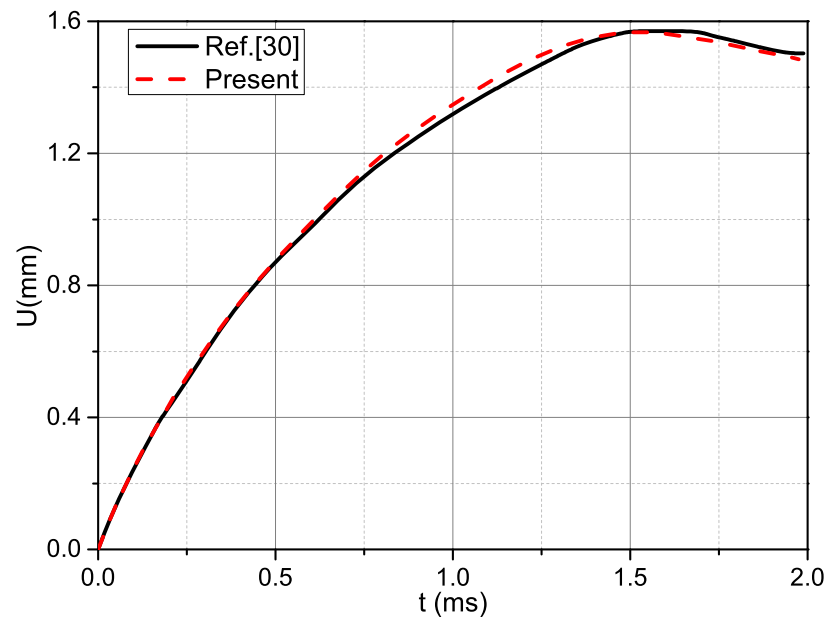

Figure 4. Comparison of the centre displacement with the results obtained from Ref. [30].

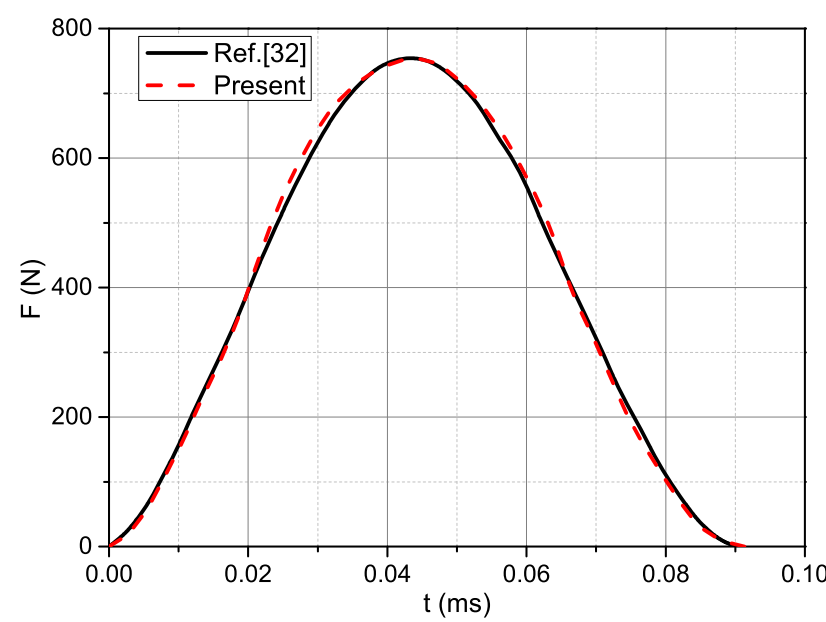

Figure 5. Comparison of the centre displacement with the results obtained from Ref. [32].

Table 1. Material properties of FG-CNTRC matrix [24].

\begin{tabular}{ll}
\hline$E^{m}[\mathrm{GPa}]$ & $3.52-0.0034(300 \mathrm{~K}+\Delta T)$ \\
$v^{m}$ & 0.34 \\
$\rho^{m}\left[\mathrm{~g} / \mathrm{cm}^{3}\right]$ & 1.19 \\
$\alpha^{m}[/ \mathrm{K}]$ & $45(1+0.0005-\Delta T) \times 10^{-6}$ \\
\hline
\end{tabular}

Table 2. Material properties of sandwich panel core [28].

\begin{tabular}{llll}
\hline$E^{f}[\mathrm{GPa}]$ & $v^{f}$ & $\rho^{f}\left[\mathrm{~g} / \mathrm{cm}^{3}\right]$ & $\alpha^{f}[/ \mathrm{K}]$ \\
$689 \mathrm{E}-3$ & 0 & 0.097 & $30 \mathrm{E}-6$ \\
\hline
\end{tabular}

Table 3. Material properties for the $(10,10)$ SWCNT [24].

\begin{tabular}{llllll}
\hline$T[\mathrm{~K}]$ & $E_{11}^{c n}[\mathrm{GPa}]$ & $E_{22}^{c n}[\mathrm{GPa}]$ & $G_{12}^{c n}[\mathrm{GPa}]$ & $v_{12}^{c n}$ & $\rho^{c n}$ \\
300 & 5.6466 & 7.0800 & 1.9445 & 0.175 & 2.1 \\
500 & 5.5308 & 6.9348 & 1.9643 & 0.175 & 2.1 \\
700 & 5.4744 & 6.8641 & 1.9644 & 0.175 & 2.1 \\
\hline
\end{tabular}

Table 4. Efficiency parameters of CNTRC for various volume fractions of CNT [24].

\begin{tabular}{llll}
\hline$V_{*}^{c n}$ & 0.12 & 0.17 & 0.28 \\
$\eta_{1}$ & 0.137 & 0.142 & 0.141 \\
$\eta_{2}$ & 1.022 & 1.626 & 1.585 \\
$\eta_{3}$ & 0.715 & 1.138 & 1.109 \\
\hline
\end{tabular}
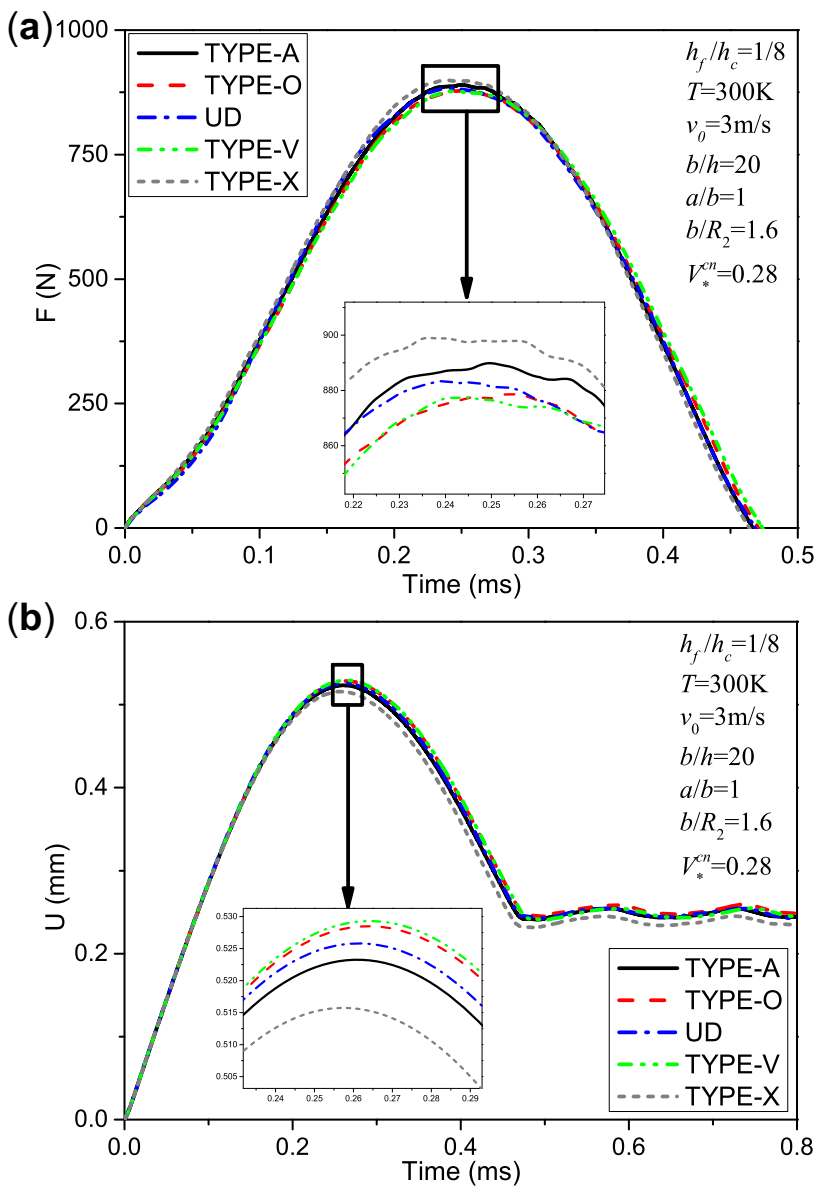

Figure 6. Impact response of different gradient forms of FGCNTRC sandwich panels: (a) contact force and (b) panel centre displacement. 

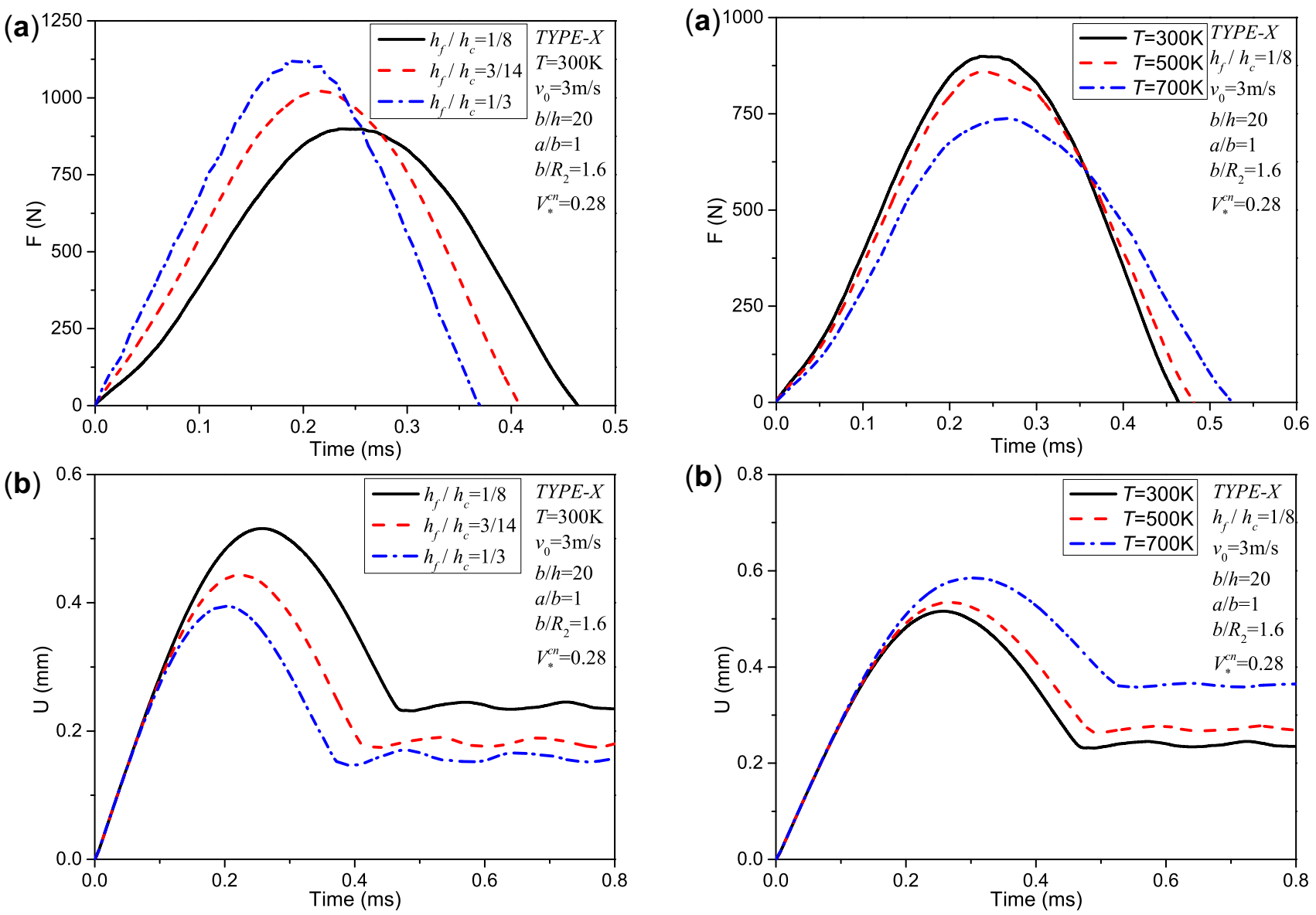

Figure 7. Impact response of different relative thicknesses of FG-CNTRC sandwich panels: (a) contact force and (b) panel centre displacement.

At $X=0, a: \bar{W}=\bar{\Psi}_{y}=0, \bar{M}_{x}=\bar{P}_{x}=0, \int_{0}^{b} \bar{N}_{x} d Y=0$.

At $Y=0, b: \bar{W}=\bar{\Psi}_{x}=0, \bar{M}_{y}=\bar{P}_{y}=0, \int_{0}^{a} \bar{N}_{y} d X=0$.

\subsection{Impact load and solution procedure}

The sandwich panel impacted by a steel ball at a rather low speed has been considered in the study. In accordance with the Hertz contact law, the contact force can be depicted as [37]

$$
P_{c}(t)=K_{c} \delta(t)^{\frac{3}{2}}=K_{c}\left[W_{0}-W\left(\frac{a}{2}, \frac{b}{2}, t\right)\right]^{\frac{3}{2}}
$$

where $P_{c}$ refers to the contact force and $\delta$ refers to the panel's indentation. $W_{0}$ and $W\left(\frac{a}{2}, \frac{b}{2}, t\right)$ refer, respectively, to the displacement of the force-acting point of the impactor and the panel presented in figure $3 . K_{c}$ refers to the modified Hertz contact stiffness that can be obtained as [36]

Figure 8. Impact response of different temperatures of FGCNTRC sandwich panels: (a) contact force and (b) panel centre displacement.

$$
K_{c}=\frac{4}{3}\left[\sqrt{\frac{R_{p} R_{i}}{R_{p}+R_{i}}} /\left(\frac{1-v_{12}}{E_{i}}+\frac{1}{E_{p}}\right)\right]
$$

where $E_{i}$ and $E_{p}$ are the elastic moduli; $R_{i}$ and $R_{p}$ are the radii, while $v_{12}$ refers to the Poisson's ratio of the panel. The panel is denoted by the subscript $p$ and the impactor is denoted by $i$. $E_{p}$ is the transverse elastic modulus at the top surface of the panel, which can be approximated to be the same as $E_{22}$ of Eq. (1b) $[32,35]$. The impactor's displacement can be zero before the contact. $W_{0}$ can be obtained as

$$
W_{0}=v_{0} t-\frac{1}{m_{i}} \int_{0}^{t} P_{c}(\tau)(t-\tau) d \tau
$$

where $m_{i}$ refers to the impactor's mass. Putting Eq. (14) and (15) into (13), we can obtain the contact equations as

$$
\left(\frac{P_{c}(t)}{K_{c}}\right)^{\frac{2}{3}}=v_{0} t-\frac{1}{m_{i}} \int_{0}^{t} P_{c}(\tau)(t-\tau) d \tau-W\left(\frac{a}{2}, \frac{b}{2}, t\right)
$$

A two-step perturbation method similar to that mentioned in Ref. [34] was used to solve the governing 

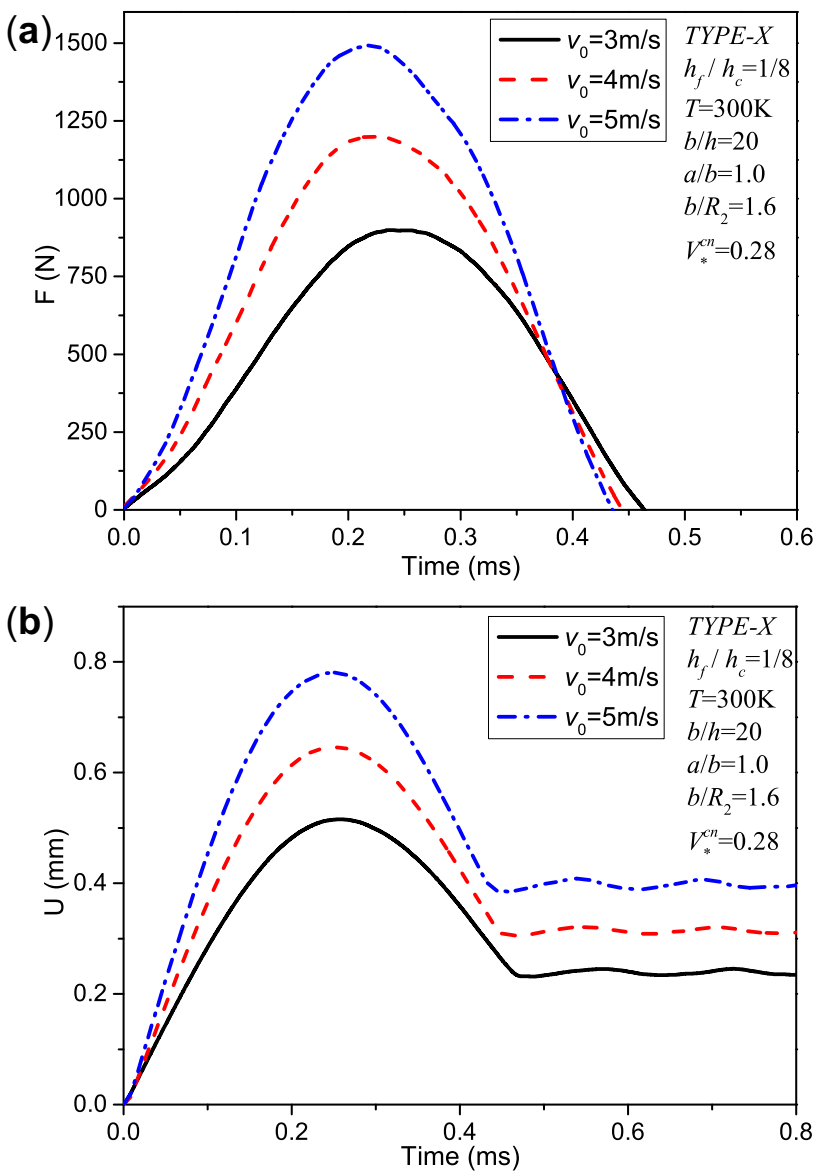

Figure 9. Impact response of different initial impact velocities of FG-CNTRC sandwich panels: (a) contact force and (b) panel centre displacement.

equations of the FG-CNTRC sandwich panel. The dimensionless governing equations and boundary conditions can be obtained using the earlier non-dimensional quantities. The solutions can be expressed as

$$
\begin{aligned}
& W(x, y, \tau)=W^{*}(x, y)+\widetilde{W}(x, y, \tau) \\
& \psi_{x}(x, y, \tau)=\psi_{x}^{*}(x, y)+\widetilde{\psi}_{x}(x, y, \tau) \\
& \psi_{y}(x, y, \tau)=\psi_{y}^{*}(x, y)+\widetilde{\psi}_{y}(x, y, \tau)
\end{aligned}
$$

where the superscript $*$ refers to the initial part, which can be written in a form similar to that in Ref. [38]. The $\sim$ symbol refers to the additional part, which can be written in a form similar to that in Ref. [34]. The displacement of the sandwich panel can be obtained using the Runge-Kutta iteration.

\section{Results and discussion}

\subsection{Comparison studies}

The FG-CNTRC plate's low-velocity impact analysis has been performed in Ref. [30]. The shape, size of the plate and the
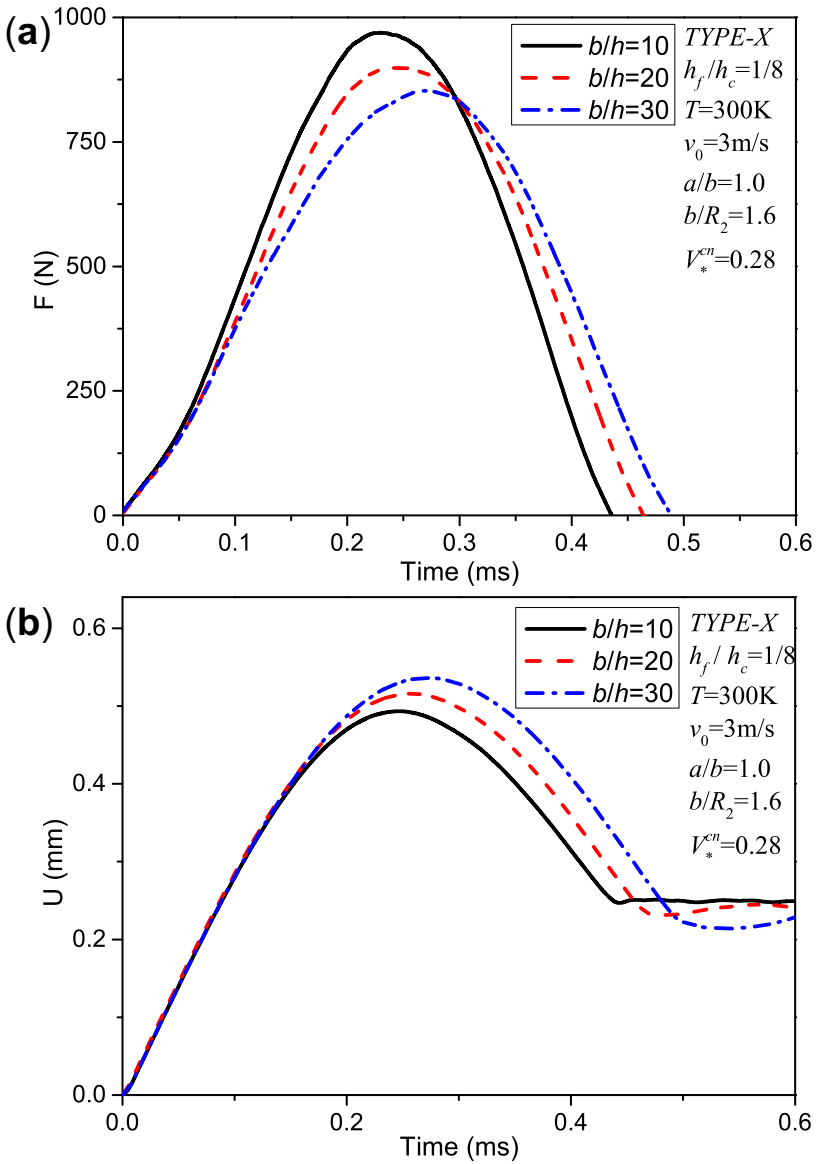

Figure 10. Impact response of different $b / h$ of FG-CNTRC sandwich panels: (a) contact force and (b) panel centre displacement.

thermal physical parameters mentioned within the reference are adopted for validating the computing pattern's accuracy within the paper. The plate consists of a single-layer FG-CNTRC of thickness $0.01 \mathrm{~m}$, width $1 \mathrm{~m}$, length $1 \mathrm{~m}$, gradient model UD and volume fraction $V_{*}^{c n}=0.28$. The temperature was set as $T=300 \mathrm{~K}$. The impactor was set as a steel ball with the primary impact velocity $v_{0}=3 \mathrm{~m} / \mathrm{s}$, mass $m_{i}=0.5 \mathrm{~kg}$ and radius $R_{i}=0.25 \mathrm{~m}$. By comparing with the outcomes in Ref. [30], the plate centre displacement-time history curves are presented in figure 4. The shell consists of single-layer FG-CNTRC of thickness $5 \mathrm{~mm}$ and radius $10 \mathrm{~mm}$. The gradient form was TYPE-X, temperature $T=300 \mathrm{~K}$ and volume fraction $V_{*}^{c n}=0.28$. The impactor was set as the reference mentioned. By comparing with the outcomes in Ref. [32], the contact force is presented in figure 5 and the results are in good agreement.

\subsection{Comparison studies}

After the verification of the numerical model, FG-CNTRC curved panel's low-velocity impact response at various gradient forms, relative thickness, temperature, CNT volume fraction and structure size as well as initial impact 

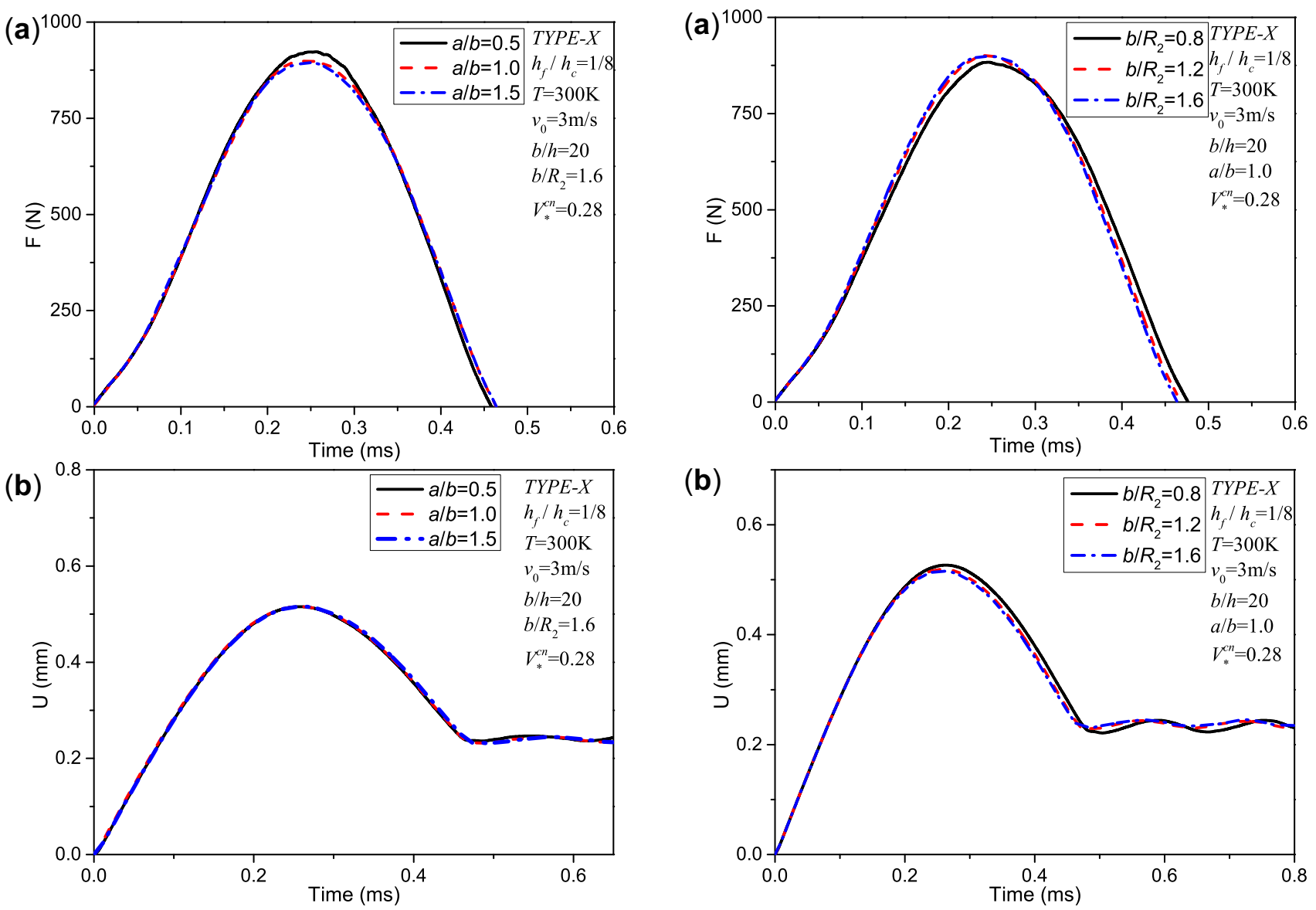

Figure. 11. Impact response of different $a / b$ of FG-CNTRC sandwich panels: (a) contact force and (b) panel centre displacement.

velocity has been explored. PMMA is considered for the matrix of FG-CNTRC. The material properties of PMMA are listed in table 1 [24]. The material properties of the sandwich panel core are listed in table 2 [28]. The material properties of CNTs mentioned in this paper are presented in table 3 [24] with $G_{13}^{c n}=G_{12}^{c n}$. The CNT's efficiency parameters are given in table 4 [24].

Typical results are shown in figures $6-13$. The overall dimensions of the sandwich panel are $h_{f} / h_{c}=1 / 8$, $b / h=20, a / b=1, a / R_{1}=1.6$ and $b / R_{2}=1.6$. The impactor's radius has been $R_{i}=10 \mathrm{~mm}$. The volume fraction, impact velocity, temperature and gradient form under the primary condition were, respectively, set to $V_{*}^{c n}=0.28$, $v_{0}=3 \mathrm{~m} / \mathrm{s}, T=300 \mathrm{~K}$ and TYPE-X.

The low-velocity impact response of FG-CNTRC curved panels of five gradient types is presented in figure 6. From small to large on contact force in order was TYPE-V, TYPE-O, UD, TYPE-A and TYPE-X. The contact time decreased with the increase of contact force. It can be inferred that the stiffness of the non-contact surface has a large influence on contact force. Type TYPE-O and TYPE$\mathrm{V}$ had better cushion performance than other types. From small to large on the panel surface, centre's displacement in

Figure 12. Impact response of different $b / R_{2}$ of FG-CNTRC sandwich panels: (a) contact force and (b) panel centre displacement.

order was TYPE-X, TYPE-A, UD, TYPE-O and TYPE-V. It implies that type TYPE-X and TYPE-A have larger impact stiffness. Hence, various types of FG-CNTRC could be used for different operating conditions providing stiffness or cushion performance.

The low-velocity impact response due to various thicknesses of FG-CNTRC layer has been shown in figure 7. It shows that increase of FG-CNTRC layer's thickness results in smaller panel centre's displacement, shorter contact time and larger contact force. All the results matched the material property of FG-CNTRC and core layer.

Figure 8 presents the impact response of FG-CNTRC at three temperatures. The results show that increasing the temperature of panel results in smaller contact force, longer contact time and larger displacement of panel centre. It can be inferred that increasing temperature has a large effect on the stiffness of FG-CNTRC structure. Both CNTs and PMMA went soft at high temperatures. In contrast, the response change was more obvious with the temperature variation from 500 to $700 \mathrm{~K}$.

FG-CNTRC curved panels' low-velocity impact response at various initial impact velocities is shown in figure 9. It can be seen that increasing initial impact 


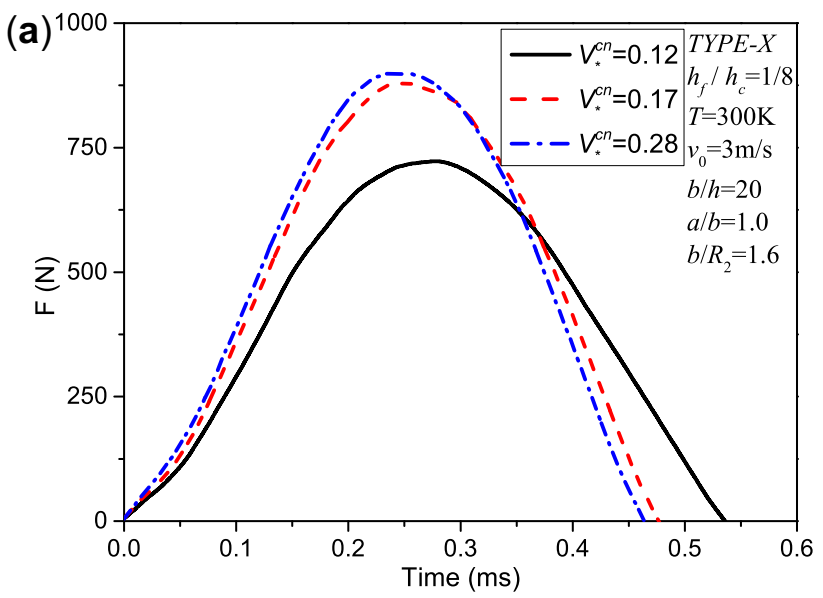

(b)

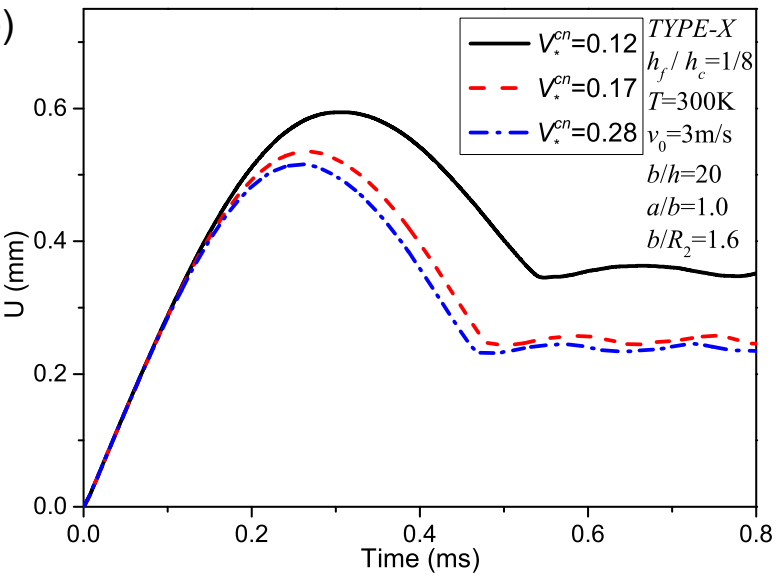

Figure 13. Impact response of different CNT volume fractions of FG-CNTRC sandwich panels: (a) contact force and (b) panel centre displacement.

velocity results in larger contact force, shorter contact time, larger displacement of panel centre and larger rate of deformation. The initial impact velocity has larger influence on FG-CNTRC curved panel's dynamic response, compared with temperature.

The low-velocity impact response of FG-CNTRC curved panel of various panel side-to-thickness ratios $b / h$ has been presented in figure 10. It could be seen that the contact force decreased while contact time and displacement of panel centre increased with increase in $b / h$. The influence of panel aspect ratio $a / b$ is presented in figure 11 . The results show that $a /$ $b$ affects the dynamic response less. The contact force was larger and contact time was shorter when $a / b=0.5$, while the displacements of panel centre of three conditions were nearly the same. Figure 12 shows the influence panel curvature ratio $b / R_{2}$ on FG-CNTRC panel's impact response. The contact force was smaller, contact time was longer and the displacement of panel centre was larger when $b / R_{2}=0.8$.

Three types of CNTs' volume fractions mentioned in table 2 had been considered for investigating FG-CNTRC panels' impact response of figure 13. The increase in CNTs' volume fraction could cause smaller panel centre displacement, shorter contact time and bigger contact force. It implies that enhanced volume fraction of CNTs could nonlinearly increase the stiffness of panel structure. The response changed with the volume fraction is more obvious from 0.12 to 0.17 .

\section{Conclusion}

The dynamic response of the FG-CNTRC sandwich curved panels under low-velocity impact load has been put forward and the panel centre's displacement and contact behaviours are explored. FG-CNTRC's material features are set in accordance with the rule of mixture. PMMA and CNTs' parameters are temperature-dependent. The von-Kármántype large-deflection equations are adopted for establishing the curved panel's nonlinear governing equations. The Hertz contact law has been set up for obtaining the contact response between the impactor and the panel.

From small to large, the five types of FG-CNTRC on contact force in order are TYPE-V, TYPE-O, UD, TYPE-A and TYPE-X, and the panel surface centre's displacement is TYPE-X, TYPE-A, UD, TYPE-O and TYPE-V from small to large. The stiffness of the non-contact surface has large influence on contact force. Other working conditions show that the low-velocity impact response of FG-CNTRC panel is significantly influenced by relative thickness, temperature, initial impact velocity, panel side to thickness ratio and CNT volume fraction. The panel aspect ratio and panel curvature ratio affected the low-velocity impact response less.

\section{Acknowledgements}

The authors would gratefully acknowledge the financial support from the Natural Science Funds for Young Scholar of Jiangsu Province (No. BK 20170837) and the fundamental research funds for the central universities (No. 309181B8807).

\section{Appendix I.}

$$
\begin{aligned}
L_{11}()= & \frac{4}{3 h^{2}}\left[F_{11}^{*} \frac{\partial^{4}}{\partial X^{4}}+2\left(F_{16}^{*}+F_{61}^{*}\right) \frac{\partial^{4}}{\partial X^{3} \partial Y}\right. \\
& +\left(F_{12}^{*}+F_{21}^{*}+4 F_{66}^{*}\right) \frac{\partial^{4}}{\partial X^{2} \partial Y^{2}} \\
& \left.+2\left(F_{26}^{*}+F_{62}^{*}\right) \frac{\partial^{4}}{\partial X \partial Y^{3}}+F_{22}^{*} \frac{\partial^{4}}{\partial Y^{4}}\right], \\
L_{12}()= & {\left[D_{11}^{*}-\frac{4}{3 h^{2}} F_{11}^{*}\right] \frac{\partial^{3}}{\partial X^{3}} } \\
& +\left[3 D_{16}^{*}-\frac{4}{3 h^{2}}\left(F_{61}^{*}+2 F_{16}\right)\right] \frac{\partial^{3}}{\partial X^{2} \partial Y}
\end{aligned}
$$




$$
\begin{aligned}
& +\left[\left(D_{12}^{*}+2 D_{66}^{*}\right)-\frac{4}{3 h^{2}}\left(F_{12}^{*}+2 F_{66}^{*}\right)\right] \frac{\partial^{3}}{\partial X \partial Y^{2}} \\
& +\left[D_{26}^{*}-\frac{4}{3 h^{2}} F_{26}\right] \frac{\partial^{3}}{\partial Y^{3}}, \\
& L_{13}()=\left[D_{16}^{*}-\frac{4}{3 h^{2}} F_{16}^{*}\right] \frac{\partial^{3}}{\partial X^{3}} \\
& +\left[\left(D_{12}^{*}+2 D_{66}^{*}\right)-\frac{4}{3 h^{2}}\left(F_{21}^{*}+2 F_{66}^{*}\right)\right] \frac{\partial^{3}}{\partial X^{2} \partial Y} \\
& +\left[3 D_{26}^{*}-\frac{4}{3 h^{2}}\left(F_{62}^{*}+2 F_{26}^{*}\right)\right] \frac{\partial^{3}}{\partial X \partial Y^{2}} \\
& +\left[D_{22}^{*}-\frac{4}{3 h^{2}} E_{22}^{*}\right] \frac{\partial^{3}}{\partial Y^{3}}, \\
& L_{14}()=B_{21}^{*} \frac{\partial^{4}}{\partial X^{4}}+\left(2 B_{26}^{*}-B_{61}^{*}\right) \frac{\partial^{4}}{\partial X^{3} \partial Y} \\
& +\left(B_{11}^{*}+B_{22}^{*}-2 B_{66}^{*}\right) \frac{\partial^{4}}{\partial X^{2} \partial Y^{2}} \\
& +\left(2 B_{16}^{*}-B_{62}^{*}\right) \frac{\partial^{4}}{\partial X \partial Y^{3}}+B_{12}^{*} \frac{\partial^{4}}{\partial Y^{4}}, \\
& L_{15}\left(\bar{N}^{T}\right)=\frac{\partial^{2}}{\partial X^{2}}\left(B_{11}^{*} \bar{N}_{x}^{T}+B_{21}^{*} \bar{N}_{y}^{T}\right)+2 \frac{\partial^{2}}{\partial X \partial Y}\left(B_{b 6}^{*} \bar{N}_{x y}^{T}\right) \\
& +\frac{\partial^{2}}{\partial Y^{2}}\left(B_{12}^{*} \bar{N}_{x}^{T}+B_{22}^{*} \bar{N}_{y}^{T}\right), \\
& L_{16}\left(\bar{M}^{T}\right)=\frac{\partial^{2}}{\partial X^{2}}\left(\bar{M}_{x}^{T}\right)+2 \frac{\partial^{2}}{\partial X \partial Y}\left(\bar{M}_{x y}^{T}\right)+\frac{\partial^{2}}{\partial Y^{2}}\left(\bar{M}_{y}^{T}\right) \text {, } \\
& L_{21}()=A_{22}^{*} \frac{\partial^{4}}{\partial X^{4}}-2 A_{26}^{*} \frac{\partial^{4}}{\partial X^{3} \partial Y}+\left(2 A_{12}^{*}+A_{66}^{*}\right) \frac{\partial^{4}}{\partial X^{2} \partial Y^{2}} \\
& +2 A_{16}^{*} \frac{\partial^{4}}{\partial X \partial Y^{3}}+A_{11}^{*} \frac{\partial^{4}}{\partial Y^{4}}, \\
& L_{22}()=\left[B_{21}^{*}-\frac{4}{3 h^{2}} E_{21}^{*}\right] \frac{\partial^{3}}{\partial X^{3}} \\
& +\left[B_{26}^{*}-B_{61}^{*}-\frac{4}{3 h^{2}}\left(E_{26}^{*}-E_{61}^{*}\right)\right] \frac{\partial^{3}}{\partial X^{2} \partial Y} \\
& +\left[B_{11}^{*}-B_{66}^{*}-\frac{4}{3 h^{2}}\left(E_{11}^{*}-E_{66}^{*}\right)\right] \frac{\partial^{3}}{\partial X \partial Y^{2}} \\
& +\left[B_{16}^{*}-\frac{4}{3 h^{2}} E_{16}^{*}\right] \frac{\partial^{3}}{\partial Y^{3}} \\
& L_{23}()=\left[B_{26}^{*}-\frac{4}{3 h^{2}} E_{26}^{*}\right] \frac{\partial^{3}}{\partial X^{3}} \\
& +\left[B_{22}^{*}-B_{66}^{*}-\frac{4}{3 h^{2}}\left(E_{22}^{*}-E_{66}^{*}\right)\right] \frac{\partial^{3}}{\partial X^{2} \partial Y} \\
& +\left[B_{16}^{*}-B_{62}^{*}-\frac{4}{3 h^{2}}\left(E_{16}^{*}-E_{62}^{*}\right)\right] \frac{\partial^{3}}{\partial X \partial Y^{2}} \\
& +\left[B_{12}^{*}-\frac{4}{3 h^{2}} E_{12}^{*}\right] \frac{\partial^{3}}{\partial Y^{3}},
\end{aligned}
$$

$$
\begin{aligned}
& L_{24}()=\frac{4}{3 h^{2}}\left[E_{21}^{*} \frac{\partial^{4}}{\partial X^{4}}+\left(2 E_{26}^{*}-E_{61}^{*}\right) \frac{\partial^{4}}{\partial X^{3} \partial Y}\right. \\
& +\left(E_{11}^{*}+E_{22}^{*}-2 E_{66}^{*}\right) \frac{\partial^{4}}{\partial X^{2} \partial Y^{2}} \\
& \left.+\left(2 E_{16}^{*}-E_{62}^{*}\right) \frac{\partial^{4}}{\partial X \partial Y^{3}}+E_{12}^{*} \frac{\partial^{4}}{\partial Y^{4}},\right] \\
& L_{25}\left(\bar{N}^{T}\right)=\frac{\partial^{2}}{\partial X^{2}}\left(A_{12}^{*} \bar{N}_{x}^{T}+A_{22}^{*} \bar{N}_{y}^{T}\right)-\frac{\partial^{2}}{\partial X \partial Y}\left(A_{66}^{*} \bar{N}_{x y}^{T}\right) \\
& +\frac{\partial^{2}}{\partial Y^{2}}\left(A_{11}^{*} \bar{N}_{x}^{T}+A_{12}^{*} \bar{N}_{y}^{T}\right), \\
& L_{31}()=\left[A_{55}-\frac{8}{h^{2}} D_{55}+\frac{16}{h^{4}} F_{55}\right] \frac{\partial}{\partial X} \\
& +\left[A_{45}-\frac{8}{h^{2}} D_{45}+\frac{16}{h^{4}} F_{45}\right] \frac{\partial}{\partial Y} \\
& +\frac{4}{3 h^{2}}\left[\left(F_{11}^{*}-\frac{4}{3 h^{2}} H_{11}^{*}\right) \frac{\partial^{3}}{\partial X^{3}}\right. \\
& +\left(F_{16}^{*}+2 F_{61}^{*}-\frac{4}{h^{2}} H_{16}^{*}\right) \frac{\partial^{3}}{\partial X^{2} \partial Y} \\
& +\left(F_{21}^{*}+2 F_{66}^{*}-\frac{4}{3 h^{2}}\left(H_{12}^{*}+2 H_{66}^{*}\right)\right) \frac{\partial^{3}}{\partial X \partial Y^{2}} \\
& \left.+\left(F_{26}^{*}-\frac{4}{3 h^{2}} H_{26}^{*}\right) \frac{\partial^{3}}{\partial Y^{3}}\right] \text {, } \\
& L_{32}()=\left[A_{55}-\frac{8}{h^{2}} D_{55}+\frac{16}{h^{4}} F_{55}\right] \\
& -\left[D_{11}^{*}-\frac{8}{3 h^{2}} F_{11}^{*}+\frac{16}{9 h^{4}} H_{11}^{*}\right] \frac{\partial^{2}}{\partial X^{2}} \\
& -2\left[D_{16}^{*}-\frac{4}{3 h^{2}}\left(F_{16}^{*}+F_{61}^{*}\right)+\frac{16}{9 h^{4}} H_{16}^{x}\right] \frac{\partial^{2}}{\partial X \partial Y} \\
& -\left[D_{66}^{*}-\frac{4}{3 h^{2}} F_{66}^{*}+\frac{16}{9 h^{4}} H_{66}^{*}\right] \frac{\partial^{2}}{\partial Y^{2}}, \\
& L_{33}()=\left[A_{45}-\frac{8}{h^{2}} D_{45}+\frac{16}{h^{4}} F_{45}\right] \\
& -\left[D_{16}^{*}-\frac{4}{3 h^{2}}\left(F_{16}^{*}+F_{61}^{*}\right)+\frac{16}{9 h^{4}} H_{16}^{*}\right] \frac{\partial^{2}}{\partial X^{2}} \\
& -\left[D_{12}^{*}+D_{66}^{*}-\frac{4}{3 h^{2}}\left(F_{12}^{*}+F_{21}^{*}+2 F_{66}^{*}\right)\right. \\
& \left.+\frac{16}{9 h^{4}}\left(H_{12}^{*}+H_{66}^{x}\right)\right] \frac{\partial^{2}}{\partial X \partial Y} \\
& -\left[D_{26}^{*}-\frac{4}{3 h^{2}}\left(F_{26}^{*}+F_{62}^{*}\right)+\frac{16}{9 h^{4}} H_{26}^{*}\right] \frac{\partial^{2}}{\partial Y^{2}}, \\
& L_{34}()=L_{22}(), L_{35}\left(\bar{N}^{T}\right) \\
& =\frac{\partial}{\partial X}\left[\left(B_{11}^{*}-\frac{4}{3 h^{2}} E_{11}^{*}\right) \bar{N}_{x}^{T}\right.
\end{aligned}
$$




$$
\begin{aligned}
& \left.+\left(B_{21}^{*}-\frac{4}{3 h^{2}} E_{21}^{*}\right) \bar{N}_{y}^{T}\right] \\
& +\frac{\partial}{\partial Y}\left[\left(B_{66}^{*}-\frac{4}{3 h^{2}}\right) \bar{N}_{x y}^{T}\right] \\
& L_{36}\left(\bar{S}^{T}\right)=\frac{\partial}{\partial X}\left(\bar{S}_{x}^{T}\right)+\frac{\partial}{\partial Y}\left(\bar{S}_{x y}^{T}\right), \\
& L_{41}()=\left[A_{45}-\frac{8}{h^{2}} D_{45}+\frac{16}{h^{4}} F_{45}\right] \frac{\partial}{\partial X} \\
& +\left[A_{44}-\frac{8}{h^{2}} D_{44}+\frac{16}{h^{4}} F_{44}\right] \frac{\partial}{\partial Y} \\
& +\frac{4}{3 h^{2}}\left[\left(F_{16}^{*}-\frac{4}{3 h^{2}} H_{16}^{*}\right) \frac{\partial^{3}}{\partial X^{3}}\right. \\
& +\left(F_{12}^{*}+2 F_{66}^{*}-\frac{4}{3 h^{2}}\left(H_{12}^{*}-2 H_{66}^{*}\right)\right) \frac{\partial^{3}}{\partial X^{2} \partial Y} \\
& +\left(F_{26}^{*}+2 F_{62}^{*}-\frac{4}{h^{2}} H_{26}^{*}\right) \frac{\partial^{3}}{\partial X \partial Y^{2}} \\
& \left.+\left(F_{22}^{*}-\frac{4}{3 h^{2}} H_{22}^{*}\right) \frac{\partial^{3}}{\partial Y^{3}}\right], \\
& L_{42}()=L_{33}() \text {, } \\
& L_{43}()=\left[A_{44}-\frac{8}{h^{2}} D_{44}+\frac{16}{h^{4}} F_{44}\right] \\
& -\left[D_{66}^{*}-\frac{8}{3 h^{2}} F_{66}^{*}+\frac{16}{9 h^{4}} H_{66}^{*}\right] \frac{\partial^{2}}{\partial X^{2}} \\
& -2\left[D_{26}^{*}-\frac{4}{3 h^{2}}\left(F_{26}^{*}+H_{62}^{*}\right)+\frac{16}{9 h^{4}} H_{26}^{*}\right] \frac{\partial^{2}}{\partial X \partial Y} \\
& -\left[D_{22}^{*}-\frac{8}{3 h^{2}} F_{22}^{*}+\frac{16}{9 h^{4}} H_{22}^{*}\right] \frac{\partial^{2}}{\partial Y^{2}}, \\
& L_{44}()=L_{23}(), \\
& L_{45}\left(\bar{N}^{T}\right)=\frac{\partial}{\partial X}\left[\left(B_{16}^{*}-\frac{4}{3 h^{2}} E_{16}^{*}\right) \bar{N}_{x}^{T}\right. \\
& +\left(B_{26}^{*}-\frac{4}{3 h^{2}} E_{26}^{*}\right) \bar{N}_{y}^{T} \\
& \left.+\left(B_{66}^{*}-\frac{4}{3 h^{2}} E_{66}^{*}\right) \bar{N}_{x y}^{T}\right] \\
& +\frac{\partial}{\partial Y}\left[\left(B_{12}^{*}-\frac{4}{3 h^{2}} E_{12}^{*}\right) \bar{N}_{x}^{T}\right. \\
& \left.+\left(B_{22}^{*}-\frac{4}{3 h^{2}} E_{22}^{*}\right) \bar{N}_{y}^{T}+\left(B_{62}^{*}-\frac{4}{3 h^{2}} E_{62}^{*}\right) \bar{N}_{x y}^{T}\right] \\
& L_{46}\left(\bar{S}^{T}\right)=\frac{\partial}{\partial X}\left(\bar{S}_{x y}^{T}\right)+\frac{\partial}{\partial Y}\left(\bar{S}_{y}^{T}\right), \\
& L()=\frac{\partial^{2}}{\partial X^{2}} \frac{\partial^{2}}{\partial Y^{2}}-2 \frac{\partial^{2}}{\partial X \partial Y} \frac{\partial^{2}}{\partial X \partial Y}+\frac{\partial^{2}}{\partial Y^{2}} \frac{\partial^{2}}{\partial X^{2}} .
\end{aligned}
$$

\section{References}

[1] Iijima S 2002 Carbon nanotubes: past, present, and future. Physica B: Condensed Matter 323: 1-5

[2] De Volder M F L, Tawfick S H, Baughman R H and Hart A J 2013 Carbon nanotubes: present and future commercial applications - supplemental information. Science 339: 535-539

[3] Chou T W, Gao L, Thostenson E T, Zhang Z and Byun J H 2010 An assessment of the science and technology of carbon nanotube-based fibers and composites. Composites Science and Technology 70: 1-19

[4] Moradi-Dastjerdi R, Meguid S A and Rashahmadi S 2019 Electro-dynamic analysis of smart nanoclay-reinforced plates with integrated piezoelectric layers. Applied Mathematical Modelling 75: 267-278

[5] Moradi-Dastjerdi R and Aghadavoudi F 2018 Static analysis of functionally graded nanocomposite sandwich plates reinforced by defected CNT. Composite Structures 200: 839-848

[6] Alibeigloo A 2014 Free vibration analysis of functionally graded carbon nanotube-reinforced composite cylindrical panel embedded in piezoelectric layers by using theory of elasticity. European Journal of Mechanics - A/ Solids 44: 104-115

[7] Alibeigloo A 2016 Thermoelastic analysis of functionally graded carbon nanotube reinforced composite cylindrical panel embedded in piezoelectric sensor and actuator layers. Composites Part B: Engineering 98: 225-243

[8] Shen H S and Xiang Y 2015 Thermal postbuckling of nanotube-reinforced composite cylindrical panels resting on elastic foundations. Composite Structures 123: 383-392

[9] Ansari R, Torabi J, Shojaei M F and Hasrati E 2016 Buckling analysis of axially-loaded functionally graded carbon nanotube-reinforced composite conical panels using a novel numerical variational method. Composite Structures 157: 398-411

[10] García-Macías E, Rodriguez-Tembleque L, Castro-Triguero R and Sáez A 2017 Buckling analysis of functionally graded carbon nanotube-reinforced curved panels under axial compression and shear. Composites Part B: Engineering 108: 243-256

[11] Kiani Y 2017 Dynamics of FG-CNT reinforced composite cylindrical panel subjected to moving load. Thin-Walled Structures 111: 48-57

[12] Mehri M, Asadi H and Kouchakzadeh M A 2017 Computationally efficient model for flow-induced instability of CNT reinforced functionally graded truncated conical curved panels subjected to axial compression. Computer Methods in Applied Mechanics and Engineering 318: 957-980

[13] Setoodeh A R, Shojaee M and Malekzade P H 2018 Application of transformed differential quadrature to free vibration analysis of FG-CNTRC quadrilateral spherical 
panel with piezoelectric layers. Computer Methods in Applied Mechanics and Engineering 335: 510-537

[14] Van Thanh N, Dinh Quang V, Dinh Khoa N, Seung-Eock K and Dinh Duc N 2019 Nonlinear dynamic response and vibration of FG CNTRC shear deformable circular cylindrical shell with temperature-dependent material properties and surrounded on elastic foundations. Journal of Sandwich Structures and Materials 21: 1-28

[15] Aragh B S, Barati A H and Hedayati H 2012 Eshelby-MoriTanaka approach for vibrational behavior of continuously graded carbon nanotube-reinforced cylindrical panels. Composites Part B: Engineering 43: 1943-1954

[16] Yas M H, Pourasghar A, Kamarian S and Heshmatia M 2013 Three-dimensional free vibration analysis of functionally graded nanocomposite cylindrical panels reinforced by carbon nanotube. Materials and Design 49: 583-590

[17] Shen H S and Xiang Y 2014 Nonlinear vibration of nanotube-reinforced composite cylindrical panels resting on elastic foundations in thermal environments. Composite Structures 111: 291-300

[18] Zhang L W, Lei Z X, Liew K M and Yu J L 2014 Static and dynamic of carbon nanotube reinforced functionally graded cylindrical panels. Composite Structures 111: 205-212

[19] Moradi-Dastjerdi R and Pourasghar A 2016 Dynamic analysis of functionally graded nanocomposite cylinders reinforced by wavy carbon nanotube under an impact load. Journal of Vibration and Control 22: 1062-1075

[20] Lei Z X, Zhang L W, Liew K M and Yu J L 2014 Dynamic stability analysis of carbon nanotube-reinforced functionally graded cylindrical panels using the element-free kp-Ritz method. Composite Structures 113: 328-338

[21] Lei Z X, Zhang L W and Liew K M 2015 Vibration analysis of CNT-reinforced functionally graded rotating cylindrical panels using the element-free kp-Ritz method. Composites Part B: Engineering 77: 291-303

[22] Sankar A, Natarajan S, Zineb T B and Ganapathi M 2015 Investigation of supersonic flutter of thick doubly curved sandwich panels with CNT reinforced facesheets using higherorder structural theory. Composite Structures 127: 340-355

[23] Thomas B and Roy T 2017 Vibration and damping analysis of functionally graded carbon nanotubes reinforced hybrid composite shell structures. Journal of Vibration and Control 23: $1711-1738$

[24] Shen H S and Xiang Y 2016 Postbuckling of pressure-loaded nanotube-reinforced composite doubly curved panels resting on elastic foundations in thermal environments. International Journal of Mechanical Sciences 107: 225-234

[25] Ebrahimi F and Habibi S 2018 Low-velocity impact response of laminated FG-CNT reinforced composite plates in thermal environment. Advances in Nano Research 5: 69-97
[26] Fallah M, Daneshmehr A R, Zarei H, Bisadi H and Minak G 2018 Low velocity impact modeling of functionally graded carbon nanotube reinforced composite (FG-CNTRC) plates with arbitrary geometry and general boundary conditions. Composite Structures 187: 554-565

[27] Feli S, Karami L and Jafari S S 2019 Analytical modeling of low velocity impact on carbon nanotube-reinforced composite (CNTRC) plates. Mechanics of Advanced Materials and Structures 26: 394-406

[28] Moradi-Dastjerdi R and Payganeh G 2017 Thermoelastic dynamic analysis of wavy carbon nanotube reinforced cylinders under thermal loads. Steel and Composite Structures 25: 315-326

[29] Wang Z X, Xu J and Qiao P 2014 Nonlinear low-velocity impact analysis of temperature-dependent nanotube-reinforced composite plates. Composite Structures 108: 423-434

[30] Song Z G, Zhang L W and Liew K M 2016 Dynamic responses of CNT reinforced composite plates subjected to impact loading. Composites Part B: Engineering 99: 154-161

[31] Jam J E and Kiani Y 2015 Low velocity impact response of functionally graded carbon nanotube reinforced composite beams in thermal environment. Composite Structures 132: 35-43

[32] Bayat M R, Rahmani O and Mosavi Mashhadi M 2018 Nonlinear low-velocity impact analysis of functionally graded nanotube-reinforced composite cylindrical shells in thermal environments. Polymer Composites 39: 730-745

[33] Malekzadeh P and Dehbozorgi M 2016 Low velocity impact analysis of functionally graded carbon nanotubes reinforced composite skew plates. Composite Structures 140: 728-748

[34] Yang C H, Ma W N and Ma D W 2018 Low-velocity impact analysis of carbon nanotube reinforced composite laminates. Journal of Materials Science 53: 637-656

[35] Song Z G, He X and Liew K M 2018 Dynamic responses of aerothermoelastic functionally graded CNT reinforced composite panels in supersonic airflow subjected to low-velocity impact. Composites Part B: Engineering 149: 99-109

[36] Yang C H, Ma W N, Ma D W, He Q and Zhong J L 2018 Analysis of the low velocity impact response of functionally graded carbon nanotubes reinforced composite spherical shells. Journal of Mechanical Science and Technology 32: 2681-2691

[37] Abrate S 2011 Impact engineering of composite structures. CISM Courses and Lectures 526. New York: Springer

[38] Zhang L W, Lei Z X and Liew K M 2015 An element-free IMLS-Ritz framework for buckling analysis of FG-CNT reinforced composite thick plates resting on Winkler foundations. Engineering Analysis with Boundary Elements 58: $7-17$ 